\title{
Design of Catamaran Propulsion System with Demihull Distance Variation on Hospital Ship
}

\author{
Amiadji $^{1}$, Agoes Santoso ${ }^{2}$, Bondan Al Akbar Sabastian ${ }^{3}$
}

\begin{abstract}
Ministry of Health, that limited human resources in health and treatment facilities, as well as the difficult geographical conditions causing problems transport and communication are the main problem. From the above, the problems faced by means of a mobile hospital in the form of vessels operating from one small island to another small island is the solution of health problems for the people living within the island of Madura. In this thesis the work will be done planning catamaran ship propulsion system with a variety of distances and determine the power demihull hospital equipment. From the calculation results in a variation within demihull get with $B=19.51 \mathrm{~m}, 0,1 \mathrm{~B}=\mathbf{2 1 . 4 6 , 0 , 2 B}=\mathbf{2 3 . 4 1}, 25.36$ resulting $0,3 \mathrm{~B}=97.8 \mathrm{kN}$ after the engine power of $812.71 \mathrm{~kW}$ obtained. From the selection of the engine 10 criteria obtained type Caterpillar 3508B engines. Power generators are required for a hospital ship is $75 \mathrm{~kW}$ for 4 gensets.
\end{abstract}

Keywords— catamaran, demihull, hospital ship, propulsion system, ship resistance

\section{INTRODUCTION}

A ccording to survey done by Ministry of Health, there are various problems faced by health services in the isolated area. The first problem is the limited human resources in health and treatment facilities. Thus, the difficult geographical conditions lead to transportation and communication problems.

From the problems mentioned, a hospital mobile in the form of operating vessels from one island to another small island is the solution of health problems for people living in the Madura island.

The aims of this paper are to plan catamaran propulsion system with demihull distance varieties and determine the power of hospital equipment.

\section{LITERATURE REVIEW}

Catamaran is a type of vessel which has two hull (demihulls) connected by a construction so that it becomes a unity as a single ship. Catamaran type can be divided based on the shape of the hull under water [2]

- Asymmetric Catamaran

- Symmetrical Catamaran

- Wave Piercing Catamaran

1. Twinhull ship model which both sides are symmetrical stream line (Model B)

It is ssumed as two monohull vessels in which both hulls are associated with a certain distance. Then, the

Amiadji, Department of Marine Engineering, Institut Teknologi Sepuluh Nopember, Surabaya 60111, Indonesia, Email: amiadji@yahoo.com

Agoes Santoso, Department of Marine Engineering, Institut Teknologi Sepuluh Nopember, Surabaya 60111, Indonesia, Email : agoes@its.ac.id

Bondan Al Akbar Sabastian, Departmen of Marine Engineering, Institut Teknologi Sepuluh Nopember, Surabaya 60111, Indonesia, Email : bondan.alakbar25@gmail.com system will have the same wave with a stream line shape of the vessel. Around the submerged ship in water, it will develop and produce movement. This system can be seen schematically in FIG. It may be divided into two kinds of wave, the diverging wave and transverse wave. Generally, both waves placed near the prow and stern of the ship then move forward along the hull [6].

2. Doublehull ship model which both sides are asymmetric, the outside line stream hull which straight in the inside. (Model D)

At the end of front part is the point of spreading fluid into sideways (follow the stream line) which almost the same with the image. The difference lies in the inner side walls are straight so the flow follows the shape of the hull straight up to stern ship. When it is implemented, this form will create waves to the considerable side [7].

3. Doublehull ship model which both sides are asymmetric, the inner side is stream line and straight in outside. (Model A and C)

Fluid flow is formed from the prow of the ship concentrated in the middle vessel (between two hulls) which moves into stern ship. While in the sideways, the straight flow follows the hull of outer shape until the stern as shown in picture. This model is suitable for operating vessels in rivers or places surrounded by a lot of people because the catamarans model do not cause waves to the side larger than the catamarans model which streamline as the outer.

The different between model $\mathrm{A}$ and $\mathrm{C}$ come to the deck area in each vessels [1]. Ship model $C$ has a larger deck than the ship model A.

Some advantages catamaran ship as follows:

1. Have a larger deck to carry passenger, vehicles, and large quantity goods.

2. With different hull from mono hull, this has important role to reduce the resistance on the vessel so it can produce the high speed and reduce fuel consumption.

Kinds of Propeller

Ordinary Propeller

- Fixed pitch propeller

- Controllable pitch propellers 
- Adjustable bolted propeller (Organized propeller with bolt)

1. Azzimuth thrusters [3]

2. Tunnel thrusters
3. Waterjets

4. Electrical pods

5. Voith Scneider Propeller

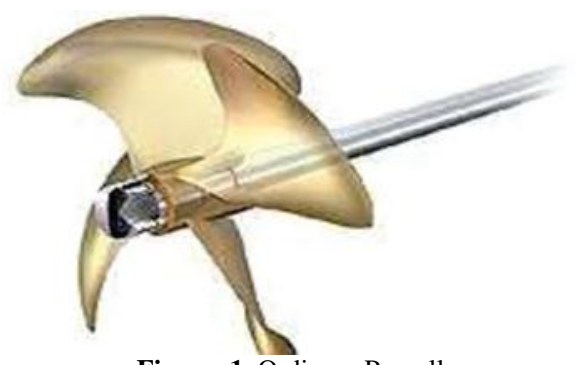

Figure. 1. Ordinary Propeller
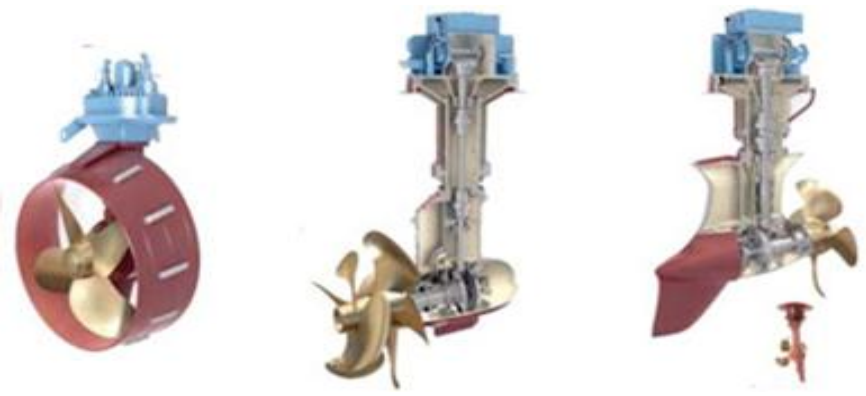

Figure. 2 Azzimuth Thrsters

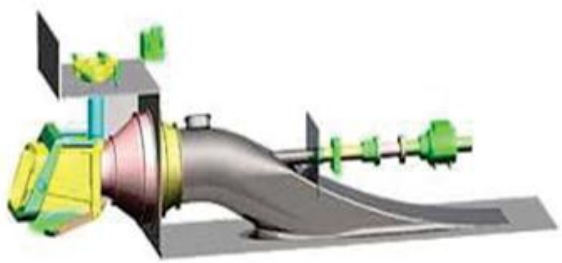

Figure. 3. Watersjets

\section{Basic Calculation Barriers}

According to Rawson and Tupper (2001), the total resistance suffered by moving ship in calm water surface consists of several components, for instance wave making resistance, skin frictional resistance, viscous pressure resistance, and water resistance resistance and appendage.

Holtrop method [4] is used to calculate the displacement full vessel obstacles in any conditions in the fixed speed remaining unchanged. The formula for calculating the barriers is:

$$
\begin{array}{ll}
\mathrm{R}_{\mathrm{T}} & =1 / 2 \times \rho \times \text { WSA } \times \mathrm{V}^{2} \times \mathrm{C}_{\mathrm{T}} \\
\mathrm{CT} & =\mathrm{C}_{\mathrm{F}}+\mathrm{C}_{\mathrm{A}}+\mathrm{CAA}+\mathrm{C}_{\mathrm{R}} \\
\mathrm{CF} & =0.075 /\left[(\text { Log Rn-2) }) \rrbracket^{2}\right. \\
\mathrm{Rn} & =(\mathrm{V} . \mathrm{Lwl}) / \mathrm{V}
\end{array}
$$

Based on the calculation of main engine [5]:

- Effective Power

$$
\text { PEservice }=\mathrm{RT}(\text { service }) \times \mathrm{Vs}
$$

- Efisiensi Propulsi

Eficiency total

$\eta \mathrm{T}=\eta \mathrm{H} . \eta \mathrm{O} . \eta \mathrm{R} . \eta \mathrm{S}$

where:

$\eta \mathrm{H}=$ Efisiensi Hull $\eta \mathrm{O}=$ Propeller in open water condition

$\eta \mathrm{R}=$ Relative rotative efficiency

$\eta \mathrm{S}=$ Transmission efficiency

- $\quad$ Trust Horse Power

THP $=\mathrm{EHP} / \eta \mathrm{H}$

The hull efficiency is the wake fraction function, w, and thrust deduction fraction, $t$, [Harvald 1983]

$\eta \mathrm{H}=(1-\mathrm{t}) /(1-\mathrm{w})$

- $\quad$ Delivery horse power (PD) $\eta \mathrm{O} . \eta \mathrm{R}$ efficiency behind propeller $\eta \mathrm{B}=\eta \mathrm{O}$. $\eta \mathrm{R} \sim \eta \mathrm{O}$ the normal amont is approximately 0,9 and 1,05

- $\quad$ Shaft Horse Power (SHP) In the planning of back side engine room location, there will be $2 \%$ lossing part while the location of the engine room on a ship midship area suffered losses of $3 \%$. It is mentioned in the book "Principal terms of Naval Architecture 131

- $\quad$ Brake Horse Power (BHP)

There is an efficiency influence of the transmission gear wheel system $(\eta \mathrm{G})$ 
- $\quad$ method of prisoners

- Holtrop Method

- Guldhammer Method

- Yamagata Method

- $\quad$ Component Prisoners That Works At The Ship In The Water

- Friction Resistance

- Residual Resistante - Wake Resistance)

- Air Resistance)

- Custody forms

- $\quad$ Added Resistance patient, care, and treatment. Generally, if it is seen from government regulation of medical devices, there are devices which are goods, apparatus, or instrument with each component of the array of parts produced and sold for maintenance, treatment, diagnosis, prevention, mitigation, and recovery for any symptoms of disorders of health that occur in the Alat bantu jalan

- Tools respirator

- Measuring instrument

- Test and laboratory equipment

- Medical Device

- Hospitals Tools

\section{Hardware General Hospital}

A completion of a required public hospital is a hospital medical equipment. Medical devices will be the means of service in public hospitals to provide action to the

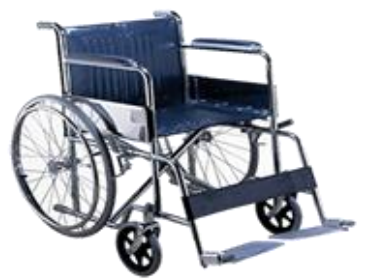

Figure. 4. Wheel chair

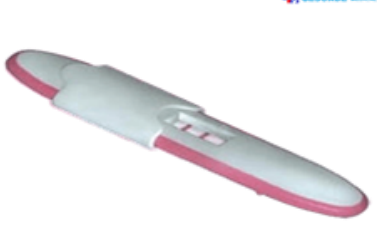

Figure. 5. Pregnancy Test Tool

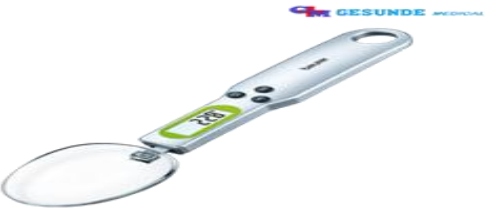

Figure. 6. tools mixer drugs

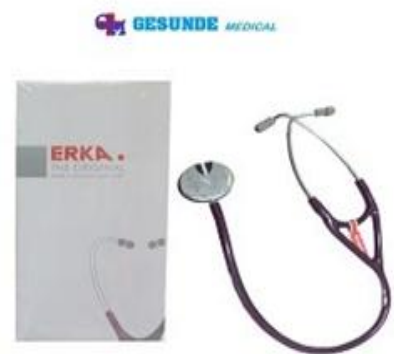

Figure. 7. Aid Measure 


\section{RESULT AND DISCUSSION}

A. Ship Dimension

$\begin{array}{ll}\text { Type } & : \text { Catamaran } \\ \text { Lpp } & : 65,00 \mathrm{~m} \\ \mathrm{~B} & : 21,46 \mathrm{~m} \\ \mathrm{H} & : 10 \mathrm{~m}\end{array}$

$$
\begin{array}{ll}
\mathrm{T} & : 3,5 \mathrm{~m} \\
\text { Rute } & : \text { Raas and Kangean Island }
\end{array}
$$

- $\quad$ Prisoners Data

By using the software maxsurf dongle resistance, at a speed of 14 knots is produced by prisoners as follows

TABLE 1

SHIP RESISTANCE

\begin{tabular}{cccccccc}
\hline \multirow{2}{*}{ Speed } & \multirow{2}{*}{ Fn } & \multirow{6}{*}{ Fn Vol } & \multicolumn{5}{c}{ Resistance } \\
\cline { 3 - 7 } & & & $0.11 \mathrm{~B}$ & $0.12 \mathrm{~B}$ & $0.13 \mathrm{~B}$ & $0.14 \mathrm{~B}$ & $0.15 \mathrm{~B}$ \\
\hline \multirow{3}{*}{14} & \multirow{3}{*}{0.292} & 0.75 & 98.3 & 98.3 & 98.2 & 98.3 & 98.3 \\
\cline { 3 - 7 } & & & $0.16 \mathrm{~B}$ & $0.17 \mathrm{~B}$ & $0.18 \mathrm{~B}$ & $0.19 \mathrm{~B}$ & $0.2 \mathrm{~B}$ \\
\cline { 3 - 7 } & & 98.3 & 98.4 & 98.4 & 98.5 & 98.5 \\
\hline
\end{tabular}

wheel system $(\eta \mathrm{G})$, on this assignment system uses a single reduction gear or single reduction gears with a loss of $2 \%$ for the forward direction so $\eta \mathrm{G}=$ $98 \%$.

1. Calculating the Effective Power Boats

Effective Power or EHP is the power required to drive the boat in the water or to pull the boat with a speed v. Calculation of the effective power vessel.

$$
\begin{aligned}
\mathrm{EHP} & =\text { Rtdinas } \times \mathrm{Vs} \\
& =113,05 \times 7,202 \\
& =814,18 \mathrm{~kW}
\end{aligned}
$$

Calculate Power on the stern tube Propeller Shafts $\mathrm{DHP}=\mathrm{EHP} / \mathrm{PC}, \mathrm{PC}=\eta \mathrm{H} \times \eta \mathrm{rr} \times \eta \mathrm{o}$.

Hull Efficiency and Wake Friction (w)

$$
\begin{aligned}
\eta \mathrm{H} & =(1-\mathrm{t}) /(1-\mathrm{w}) \\
\mathrm{w} & =0,5 \mathrm{Cb}-0,05 \\
& =(0,5 \times 0,523)-0,05 \\
& =0,2115
\end{aligned}
$$

\section{Thrust Deduction Factor $(t)$}

Values $\mathrm{t}$ can be sought from the value $\mathrm{w}$ which known as that $\mathrm{t}=\mathrm{k}$. $\mathrm{w} \mathrm{k}$ values between 0.7-0.9 and taken the value of $\mathrm{k}=0.8=0,8 \times 0,2115=$ 0,169

$$
\begin{aligned}
\eta \mathrm{H} & =(1-\mathrm{t}) /(1-\mathrm{w}) \\
& =1,053
\end{aligned}
$$

\section{Rotary Relative efficiency ( $\eta \mathbf{r r}$ )}

The $\eta$ rr value for ships with a propeller-type twin screw is 0.95 to 1.0 In planning the propeller and shaft tube is taken $\eta \mathrm{rr}=1.0$

\section{Propulsion Efficiency ( $\eta \mathbf{0})$}

is open water efficiency which efficiency of the propeller at the time of open water test. The amount is between $40-70 \%$, and taken 55\%.

$$
\begin{aligned}
& \text { Coeffisien Propulsif (PC) } \\
& \begin{aligned}
\mathrm{PC} \quad & =\eta \mathrm{H} \times \eta \mathrm{rr} \times \eta \mathrm{O} \\
& =1.053 \times 1 \times 0.55 \\
& =0,5795
\end{aligned}
\end{aligned}
$$

Then, power on the propeller shaft tube is calculated from the ratio between the effective power and the propulsive coefficient:

$$
\begin{aligned}
\mathrm{DHP} & =\mathrm{EHP} / \mathrm{PC} \\
& =1404,9 \mathrm{~kW} \\
& =1910,2 \mathrm{HP}
\end{aligned}
$$

Calculate Power On Propeller Shaft

$$
\begin{aligned}
\text { SHP } & =\mathrm{DHP} / \eta \mathrm{s} \eta \mathrm{b} \\
& =1949,18 \mathrm{HP} \\
& =1453,5 \mathrm{~kW}
\end{aligned}
$$

Calculate Prime Mover Power Required

BHPscr

The influence the efficiency of the transmission gear

$$
\begin{aligned}
\text { BHPscr } & =\mathrm{SHP} / \eta \mathrm{G} \\
& =1988,96 \mathrm{HP} \\
& =1483,2 \mathrm{~kW}
\end{aligned}
$$

BHPmcr

Power is obtained when the engine is maximum. The value of the engine when the engine is taken from the state service margin between $80 \%-85 \%$. Therefore BHPmcr obtained in the following manner:

power BHPscr taken $90 \%$ BHPmcr

$$
\begin{aligned}
\text { BHPmcr } & =\text { BHPscr } / 0.90 \\
& =2209,95 \mathrm{HP} \\
& =1625,42 \mathrm{~kW} \\
& =1617,15 / 2 \\
& =812,71 \mathrm{~kW}
\end{aligned}
$$

a) Engine $\mathrm{SFOC}$

$$
\begin{aligned}
& \text { SFOC }=1-\left(\frac{\text { SFOC engine }- \text { SFOC smallest }}{\text { SFOC smallest }} \times 100 \%\right) \\
& \text { Cat } \quad=1-\left(\frac{62,6-62,6}{62,6} \times 100 \%\right)=100 \% \\
& \text { Cummins }=1-\left(\frac{64,2-62,6}{62,6} \times 100 \%\right)=91 \% \\
& \text { ABC } \quad=1-\left(\frac{65,6-62,6}{62,6} \times 100 \%\right)=88 \%
\end{aligned}
$$

Best price is IDR 20.000 .000 .000

$$
\begin{aligned}
& \text { Price } \quad=1--\left(\frac{\text { Price engine-lowest price }}{\text { lowest price }} \times 100 \%\right) \\
& \text { Cat } \quad=1-\left(\frac{5,2 B-5,2 B}{5,2 B} \times 100 \%\right)=100 \% \\
& \text { Cummins }=1-\left(\frac{5,6 B-5,2 B}{5,2 B} \times 100 \%\right)=85 \% \\
& \mathrm{ABC} \quad=1-\left(\frac{6,1 B-5,2 B}{5,2 B} \times 100 \%\right)=80 \%
\end{aligned}
$$

b) Engine dimension

$$
\begin{aligned}
& \text { Dim } \quad=1-\left(\frac{\text { mginearmension-smanisert armension }}{\text { amallest dimension }} \times 100 \%\right) \\
& \text { Cat } \quad=1-\left(\frac{3944,8-3944,8}{3944,8} \times 100 \%\right)=100 \% \\
& \text { Cummins }=1-\left(\frac{4235-3944,8}{3944,8} \times 100 \%\right)=88 \% \\
& \text { ABC } \quad=1-\left(\frac{4379-3944,8}{3944,8} \times 100 \%\right)=82 \%
\end{aligned}
$$




\section{c) Engine Weight}

$$
\begin{aligned}
& \text { Weight }=1-\left(\frac{\text { engine weight-smallest weight }}{\text { smallest weight }} \times 100 \%\right) \\
& \text { Cat } \quad=1-\left(\frac{1772,8-1772,8}{1772,8} \times 100 \%\right)=100 \% \\
& \text { Cummins }=1-\left(\frac{1889-1772,8}{1772,8} \times 100 \%\right)=84 \% \\
& \text { ABC } \quad=1-\left(\frac{1983-1772,8}{1772,8} \times 100 \%\right)=79 \%
\end{aligned}
$$

\section{d) Engine rpm}

$$
\begin{aligned}
& \mathrm{RPM} \quad=1-\left(\frac{\text { RPM engine-RPM propeller }}{\text { RPM propelley }} \times 100 \%\right) \\
& \text { Cat } \quad=1-\left(\frac{1835-363^{3} 3}{363_{\imath} 3} \times 100 \%\right)=70 \% \\
& \text { Cummins }=1-\left(\frac{1800-363,3}{363_{n} 3} \times 100 \%\right)=71 \% \\
& \mathrm{ABC} \quad=1-\left(\frac{750-363^{3} 3}{363_{\imath} 3} \times 100 \%\right)=100 \%
\end{aligned}
$$

\begin{tabular}{|c|c|c|c|}
\hline & $\mathrm{B}$ & 19.51 & $\mathrm{~m}$ \\
\hline \multirow{10}{*}{$\mathrm{B}+$} & 0.11 & 21.6561 & $\mathrm{~m}$ \\
\hline & 0.12 & 21.8512 & $\mathrm{~m}$ \\
\hline & 0.13 & 22.0463 & $\mathrm{~m}$ \\
\hline & 0.14 & 22.2414 & $\mathrm{~m}$ \\
\hline & 0.15 & 22.4365 & $\mathrm{~m}$ \\
\hline & 0.16 & 22.6316 & $\mathrm{~m}$ \\
\hline & 0.17 & 22.8267 & $\mathrm{~m}$ \\
\hline & 0.18 & 23.0218 & $\mathrm{~m}$ \\
\hline & 0.19 & 23.2169 & $\mathrm{~m}$ \\
\hline & 0.2 & 23.412 & $\mathrm{~m}$ \\
\hline
\end{tabular}

\begin{tabular}{|c|c|c|c|c|c|c|c|}
\hline \multirow{2}{*}{$\frac{\text { Criteria }}{\text { SFOC }}$} & \multirow{2}{*}{$\frac{\text { Weight }}{20 \%}$} & \multicolumn{2}{|c|}{ Caterpillar (3508B) } & \multicolumn{2}{|c|}{ Cummins (KTA 38 M1) } & \multicolumn{2}{|c|}{ ABS (8 DXC-750-100) } \\
\hline & & $100 \%$ & $20 \%$ & $91.0 \%$ & $18 \%$ & $88.0 \%$ & $18 \%$ \\
\hline Price & $15 \%$ & $95 \%$ & $14 \%$ & $85.0 \%$ & $13 \%$ & $80.0 \%$ & $12 \%$ \\
\hline Dimension & $5 \%$ & $100 \%$ & $5 \%$ & $88.0 \%$ & $4 \%$ & $82.0 \%$ & $4 \%$ \\
\hline Weight & $5 \%$ & $100 \%$ & $5 \%$ & $84.0 \%$ & $4 \%$ & $79.0 \%$ & $4 \%$ \\
\hline RPM & $15 \%$ & $70 \%$ & $11 \%$ & $71.0 \%$ & $11 \%$ & $100.0 \%$ & $15 \%$ \\
\hline Gearbox & $5 \%$ & $100 \%$ & $5 \%$ & $85.0 \%$ & $4 \%$ & $85.0 \%$ & $4 \%$ \\
\hline Power & $15 \%$ & $81 \%$ & $12 \%$ & $79.0 \%$ & $12 \%$ & $78.0 \%$ & $12 \%$ \\
\hline Fuel & $10 \%$ & $100 \%$ & $10 \%$ & $100.0 \%$ & $10 \%$ & $100.0 \%$ & $10 \%$ \\
\hline Maintainability & $5 \%$ & $100 \%$ & $5 \%$ & $100.0 \%$ & $5 \%$ & $100.0 \%$ & $5 \%$ \\
\hline \multirow[t]{2}{*}{ Reliability } & $5 \%$ & $100 \%$ & $5 \%$ & $86.0 \%$ & $4 \%$ & $75.0 \%$ & $4 \%$ \\
\hline & \multicolumn{2}{|c|}{ Total } & $92 \%$ & & $86 \%$ & & $87 \%$ \\
\hline
\end{tabular}

\section{e) Gearbox}

$$
\begin{aligned}
& \text { Engine } \quad=1-\left(\frac{\text { Gear box price-lowest Gear box price }}{\text { lowest Gear box price }} \times 10 \mathrm{C} \%\right) \\
& \text { Cat } \quad=1-\left(\frac{18 M-18 M}{18 M} \times 100 \%\right)=100 \% \\
& \text { Cummins }=1-\left(\frac{22 M-18 M}{18 M} \times 100 \%\right)=85 \% \\
& \mathrm{ABC} \quad=1-\left(\frac{22 M-18 M}{18 M} \times 100 \%\right)=85 \%
\end{aligned}
$$

TABEL 2.

VARIASION DEMIHULL

TABEL 3.

ELECTION MACHINE WITH10CRITERIA

\section{f. Power of Engine}

Power $\quad=1-\left(\frac{\text { enginepower-power requirement }}{\text { power requirement }} \times 100 \%\right)$

Cat $\quad=1-\left(\frac{960-812,71}{812,71} \times 100 \%\right)=81 \%$

Cummins $=1-\left(\frac{979-812,71}{812,71} \times 100 \%\right)=79 \%$

$\mathrm{ABC} \quad=1-\left(\frac{983-812,71}{812,71} x 100 \%\right)=78 \%$

\section{g. Fuel}

Fueled MDO and HFO engines selected then all get the same value.

\section{h. Maintainability}

This type of machine and the installation will affect the treatment affects the cost and the number of crew aboard.

\section{i. Reliability}

The more stuff there is getting cheaper conversely the few goods that the market will be more expensive because hard to find.

Then 10 criterias of assessment resulting Caterpillar engines (3508B) which meets the requirements Propeller Selection. 
International Journal of Marine Engineering Innovation and Research, Vol. 1(3), Jun. 2017. 204-212 (pISSN: 2541-5972, eISSN: 2548-1479)

TABEL 4.

PROPELLER SELECTION

\begin{tabular}{cccccccccc}
\hline Series & Ae/A0 & $1 / \mathrm{Jb}$ & $\mathrm{P} / \mathrm{Db}$ & $\eta \mathrm{b}$ & $\mathrm{A} 0$ & $\mathrm{Ae}$ & $\mathrm{Ap}$ & $\mathrm{Vr}^{2}$ & Pitch \\
\hline & 0.45 & 1.91 & 0.94 & 0.55 & 21.97 & 9.89 & 8.42 & 81.95 & 4.9732 \\
& 0.6 & 1.92 & 0.92 & 0.552 & 22.19 & 13.31 & 11.40 & 82.46 & 4.8919 \\
& 0.75 & 1.93 & 0.93 & 0.545 & 22.42 & 16.82 & 14.36 & 82.96 & 4.9698 \\
& 0.9 & 1.88 & 0.96 & 0.537 & 21.32 & 19.19 & 16.25 & 80.47 & 5.0025 \\
& 1.05 & 1.84 & 1.02 & 0.52 & 20.45 & 21.47 & 17.90 & 78.52 & 5.2067 \\
& 0.45 & 2.2 & 0.98 & 0.579 & 29.62 & 13.33 & 11.23 & 86.55 & 6.0202 \\
& 0.6 & 2.28 & 0.95 & 0.576 & 31.36 & 18.82 & 15.98 & 89.73 & 6.0042 \\
& 0.75 & 2.25 & 0.97 & 0.575 & 30.48 & 22.86 & 19.32 & 88.13 & 6.0447 \\
& 0.9 & 2.24 & 0.98 & 0.56 & 30.20 & 27.18 & 22.90 & 87.60 & 6.0781 \\
& 1.05 & 2.18 & 1.04 & 0.548 & 28.49 & 29.91 & 24.80 & 84.48 & 6.2659 \\
\hline
\end{tabular}

TABEL 5.

CAVITATION

\begin{tabular}{cccccc}
\hline Series & Ae/A0 & T & tC & s0.7R & Cavitation \\
\hline & 0.45 & 94.69 & 0.268 & 0.351 & Cavitation \\
& 0.6 & 94.69 & 0.196 & 0.348 & No Cavitaiton \\
& 0.75 & 94.69 & 0.155 & 0.345 & No Cavitation \\
& 0.9 & 94.69 & 0.141 & 0.359 & No Cavitation \\
& 1.05 & 94.69 & 0.131 & 0.372 & No Cavitation \\
\hline & 0.45 & 94.69 & 0.190 & 0.326 & Cavitation \\
& 0.6 & 94.69 & 0.129 & 0.311 & No Cavitation \\
& 0.75 & 94.69 & 0.109 & 0.318 & No Cavitation \\
& 0.9 & 94.69 & 0.092 & 0.321 & No Cavitation \\
& 1.05 & 94.69 & 0.088 & 0.337 & No Cavitation \\
\hline
\end{tabular}

TABEL 6.

THE TYPE OF PROPELLER USED

\begin{tabular}{cccccc}
\hline Propeller type & $\mathrm{Db}(\mathrm{ft})$ & $\mathrm{n}(\mathrm{rpm})$ & $\mathrm{P} / \mathrm{Db}$ & $\eta \mathrm{b}$ & Pitch \\
\hline $\mathrm{B} 5-60$ & 6.32 & 363.37 & 0.95 & 0.576 & 6.004 \\
\hline
\end{tabular}

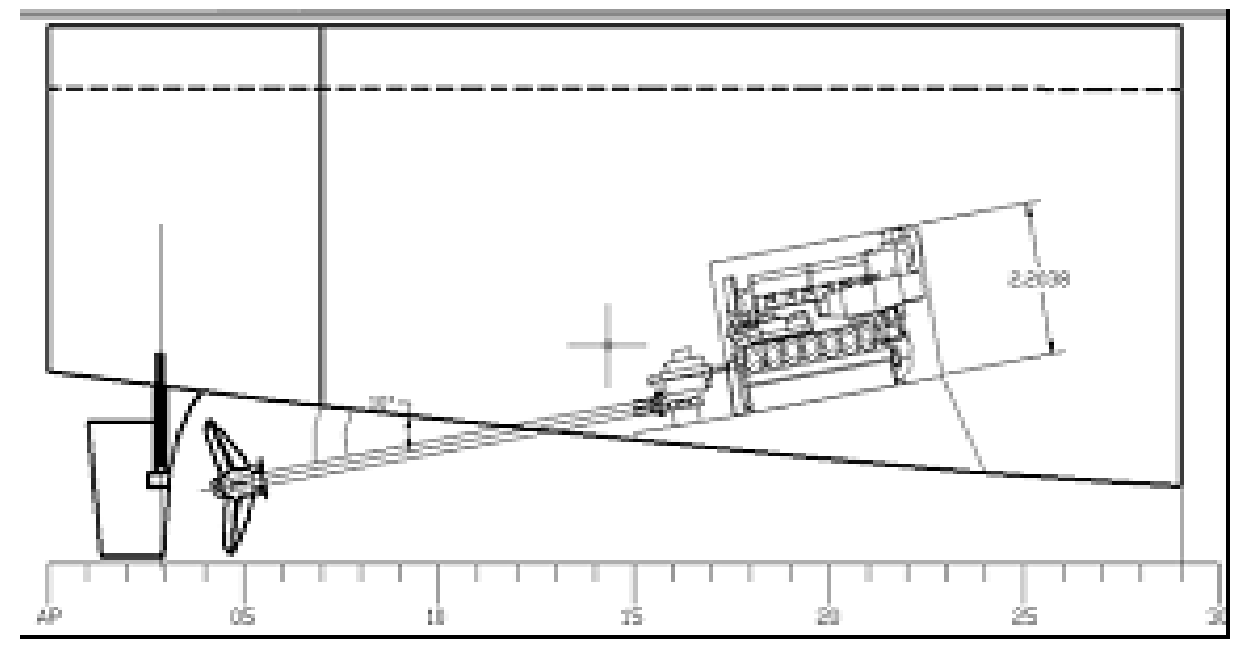

Figure. 8. The laying machine 

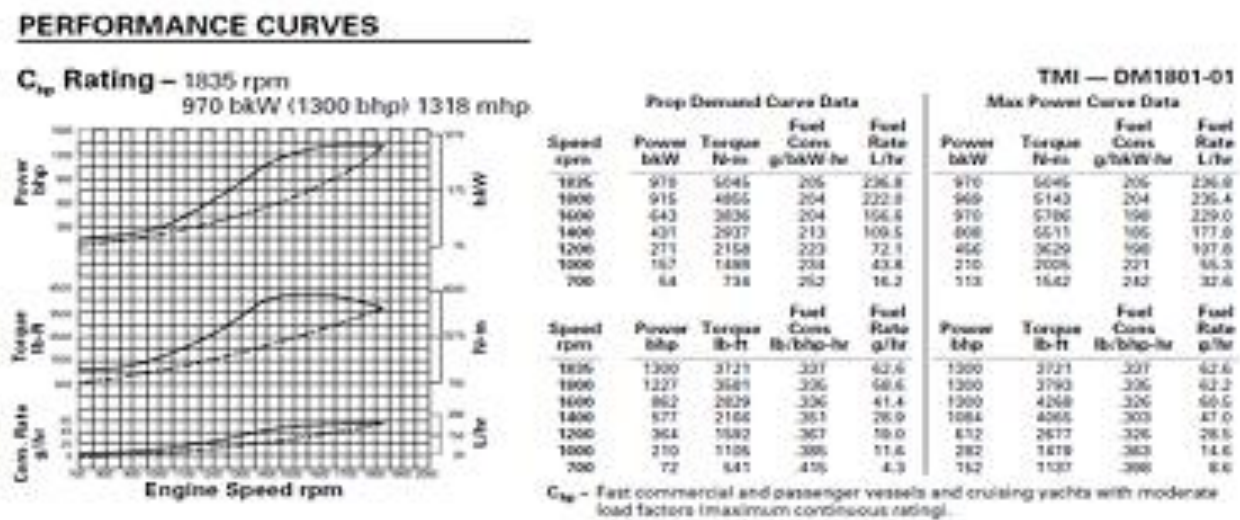

Figure. 9. The Katalog

B. Criteria Genset With 10

a. SFOC Selection

$\mathrm{SFOC}=1-\left(\frac{\text { sFoc genset-smantest SFOC }}{\text { gmallest SFOC }} \times 100 \%\right)$

Lovol $=1-\left(\frac{15,45-15,45}{15,45} \times 100 \%\right)=100 \%$

Cum $=1-\left(\frac{16-15,45}{15,45} \times 100 \%\right)=93 \%$

Deutz $=1-\left(\frac{16_{x} 5-15_{2}, 45}{15,45} \times 100 \%\right)=75 \%$

b. Best price is IDR 10.000.000.000

Lovol $=\frac{120 \mathrm{M}}{75 \mathrm{~kW}}=1,600 / \mathrm{kW}$

$\mathrm{Cum} \quad=\frac{163 \mathrm{M}}{85 \mathrm{~kW}}=1,917 / \mathrm{kW}$

Deutz $=\frac{169,2 \mathrm{M}}{88 \mathrm{~kW}}=1,922 / \mathrm{kW}$

Genset $=1-\left(\frac{\text { Genset prics/hW-lowest prices/hW }}{\text { lowest prices/hW }} \times 100 \%\right)$

Lovol $=1-\left(\frac{1,600-1,600}{1,600} \times 100 \%\right)=100 \%$

Cum $=1-\left(\frac{1,917-1,600}{1,600} \times 100 \%\right)=85 \%$

Deutz $=1-\left(\frac{1,922-1,600}{1,600} \times 100 \%\right)=81 \%$

c. Dimension

Genset $=1-\left(\frac{\text { Dimension of genset-Smairsest armension }}{\text { smallest dimension }} \times 100 \%\right)$

Lovol $=1^{-\left(\frac{4750-4750}{4750} \times 100 \%\right)}=100 \%$

Cum $=1^{-\left(\frac{4900-4750}{4750} \times 100 \%\right)}=93 \%$

Deutz $=1^{-\left(\frac{5150-4750}{4750} \times 100 \%\right)}=82 \%$

d. Weight

Genset $=1-\left(\frac{\text { weight of genset-smallest weight }}{\text { smallest weight }} \times 100 \%\right)$

Lovol $=1^{-\left(\frac{1242-1124}{1124} \times 100 \%\right)}=89 \%$

Cum $=1^{-\left(\frac{1124-1124}{1124} \times 100 \%\right)}=100 \%$

Deutz $=1^{-\left(\frac{1384-1124}{1124} \times 100 \%\right)}=86 \%$ $\begin{aligned} \text { e. } & \text { RPM } \\ \text { Genset } & =1-\left(\frac{\text { RPM genset-smallest RPM }}{\text { Rmglest } R P M} \times 100 \%\right) \\ \text { Lovol } & =1^{-\left(\frac{1500-1500}{1500} \times 100 \%\right)}=100 \% \\ \text { Cum } & =1^{-\left(\frac{1800-1500}{1500} \times 100 \%\right)}=89 \% \\ \text { Deutz } & =1^{-\left(\frac{1500-1500}{1500} \times 100 \%\right)}=100 \%\end{aligned}$

f. Frequency

Genset $=1-\left(\frac{\text { frequency of genset-frequency requirement }}{\text { frenuency requirement }} \times 100 \%\right)$

Lovol $=1^{-\left(\frac{50 \mathrm{~Hz}-50 \mathrm{~Hz}}{50 \mathrm{~Hz}} \times 100 \%\right)}=100 \%$

Cum $=1^{-\left(\frac{60 \mathrm{~Hz}-50 \mathrm{~Hz}}{50 \mathrm{~Hz}} \times 100 \%\right)}=94 \%$

Deutz $=1^{-\left(\frac{60 \mathrm{~Hz}-50 \mathrm{~Hz}}{50 \mathrm{~Hz}} \times 100 \%\right)}=94 \%$

g. Genset power

Genset $=1-\left(\frac{\text { power of genset-ponser requirement }}{\text { power requirement }} \times 100 \%\right)$

Lovol $=1^{-\left(\frac{75 \mathrm{~kW}-75 \mathrm{~kW}}{75 \mathrm{~kW}} \times 100 \%\right)}=100 \%$

Cum $=1^{-\left(\frac{85 \mathrm{~kW}-75 \mathrm{~kW}}{75 \mathrm{~kW}} \times 100 \%\right)}=91 \%$

Deutz $=1^{-\left(\frac{88 \mathrm{~kW}-75 \mathrm{~kW}}{75 \mathrm{~kW}} \times 100 \%\right)}=88 \%$

h. Fuel

Fueled HSD and MDO engines selected then all get the same value.

i. Maintainability

This type of machine and the installation will affect the treatment affects the cost and the number of crew aboard. j. Reliability

The more stuff there is getting cheaper conversely the few goods that the market will be more expensive because hard to find. 
International Journal of Marine Engineering Innovation and Research, Vol. 1(3), Jun. 2017. 204-212

TABEL 7.

SELECTION CRITERIA GENSET WITH 10 CRITERIA

\begin{tabular}{|c|c|c|c|c|c|c|c|}
\hline \multirow{2}{*}{$\begin{array}{r}\text { Criteria } \\
\text { SFOC }\end{array}$} & \multirow{2}{*}{$\frac{\text { Weight }}{20 \%}$} & \multicolumn{2}{|c|}{ Caterpillar (3508B) } & \multicolumn{2}{|c|}{ Cummins (KTA $38 \mathrm{M} 1$ ) } & \multicolumn{2}{|c|}{ ABS (8 DXC-750-100) } \\
\hline & & $100 \%$ & $20 \%$ & $91.0 \%$ & $18 \%$ & $88.0 \%$ & $18 \%$ \\
\hline Price & $15 \%$ & $95 \%$ & $14 \%$ & $85.0 \%$ & $13 \%$ & $80.0 \%$ & $12 \%$ \\
\hline Dimension & $5 \%$ & $100 \%$ & $5 \%$ & $88.0 \%$ & $4 \%$ & $82.0 \%$ & $4 \%$ \\
\hline Weight & $5 \%$ & $100 \%$ & $5 \%$ & $84.0 \%$ & $4 \%$ & $79.0 \%$ & $4 \%$ \\
\hline RPM & $15 \%$ & $70 \%$ & $11 \%$ & $71.0 \%$ & $11 \%$ & $100.0 \%$ & $15 \%$ \\
\hline Gearbox & $5 \%$ & $100 \%$ & $5 \%$ & $85.0 \%$ & $4 \%$ & $85.0 \%$ & $4 \%$ \\
\hline Power & $15 \%$ & $81 \%$ & $12 \%$ & $79.0 \%$ & $12 \%$ & $78.0 \%$ & $12 \%$ \\
\hline Fuel & $10 \%$ & $100 \%$ & $10 \%$ & $100.0 \%$ & $10 \%$ & $100.0 \%$ & $10 \%$ \\
\hline Maintainability & $5 \%$ & $100 \%$ & $5 \%$ & $100.0 \%$ & $5 \%$ & $100.0 \%$ & $5 \%$ \\
\hline Reliability & $5 \%$ & $100 \%$ & $5 \%$ & $86.0 \%$ & $4 \%$ & $75.0 \%$ & $4 \%$ \\
\hline
\end{tabular}

TABEL 8.

Genset CATALOG

\begin{tabular}{lccccc}
\hline Engine Model & Alternator model & \multicolumn{2}{c}{ Standby power } & \multicolumn{2}{c}{ Prime Power } \\
\hline \multicolumn{1}{c}{ LOVOL } & STAMORD & $\mathrm{kVA}$ & $\mathrm{kW}$ & $\mathrm{kVA}$ & $\mathrm{kW}$ \\
\hline $1004 \mathrm{G}$ & BCI184G & 34 & 27 & 31 & 25 \\
$1004 \mathrm{G}$ & $\mathrm{BCI184H}$ & 41 & 33 & 38 & 30 \\
$1004 \mathrm{G}$ & $\mathrm{BCI1} 44 \mathrm{~J}$ & 46 & 37 & 42 & 34 \\
$1004 \mathrm{G}$ & $\mathrm{UCI224D}$ & 50 & 40 & 45 & 36 \\
$1004 \mathrm{TG}$ & $\mathrm{UCI224D}$ & 55 & 44 & 50 & 40 \\
1004TG & UCI224E & 65 & 52 & 60 & 48 \\
1004TG & UCI224F & 76 & 61 & 70 & 56 \\
1006TG1A & UCI224G & 94 & 75 & 85 & 68 \\
1006TG2A & UCI274C & 110 & 88 & 100 & 80 \\
1006TAG & UCI274D & 125 & 100 & 114 & 91 \\
1006TAG & UCI274E & 150 & 120 & 135 & 108 \\
\hline
\end{tabular}
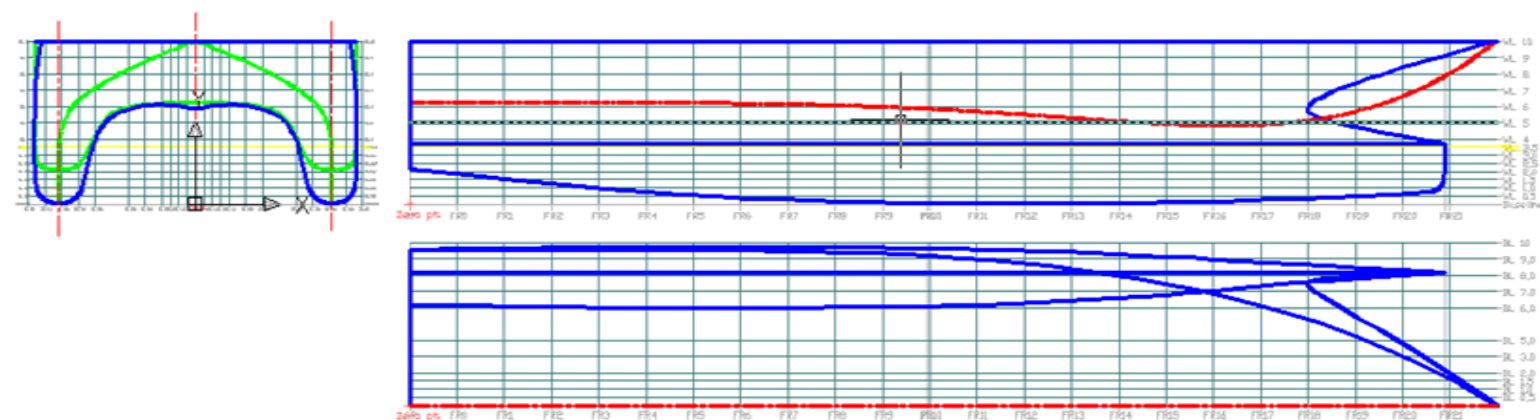

Figure. 10. Linesplan 


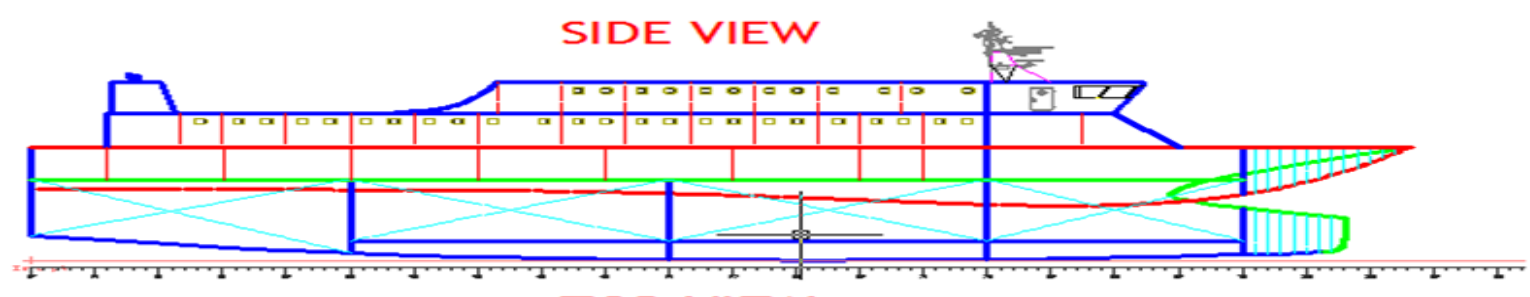

TOP VIEW

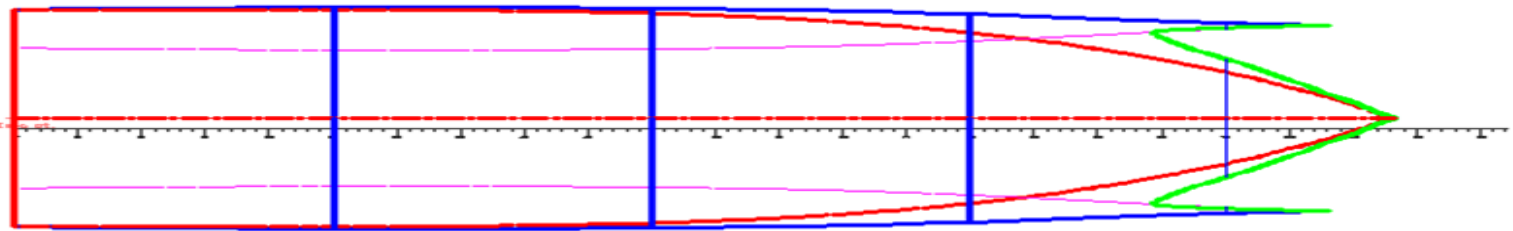

Figure. 11. General arrangement

\section{CONCLUSION}

The floating hospital ship model is obtained with these lenght in 65,00 metres, beam in 21,46 metres, max draught in 3,5 metres, speed in 14 Knots and route from Raas to Kangean Island.

This hospital ship has emergency and hospitality facilities services, surgery specialist, obstetricians and children's specialist with the total of patient's bed is 30 people.

\section{REFERENCES}

[1]. Biro Klasifikasi Indonesia. 2006. Rules for the Classification and Construction of Seagoing Steel Ship. Jakarta Edwar V. Lewis. Principles of Naval Architecture.

[2]. Lammern. Van. 1980. Resistance Propulsion and Steering of Ship. The Technical Publishing Company, H. Stam Haarlem.

[3]. Lapp. AJ, The Design of Marine Screw Propeller, 1972, Hilton Book

[4]. Sularso. Suga, Kiyokatsu. 1980. Dasar Perencanaan dan Pemilihan Elemen Mesin. PT. Pradya Paramita, Jakarta.

[5]. Widodo Adji, Suryo. Engine Propeller Matching Prosedure, 1999, Teknik Sistem Perkapalan, Surabaya.

[6]. Couser, P., Molland, A.F., Armstrong, N.a,Utama, I.K.A.P., Calm Water Powering Predictions For High Speed Catamarans, Preceeding Of FAST 97 Conference, Sydney (Australia).

[7] I. R. Kusuma, S. Sarwito, and R. A. W. A. Irawati, "Analysis of Electric Propulsion Performance on Submersible with Motor DC, Supply Power 10260AH at Voltage 115VDC," Int. J. Mar. Eng. Innov. Res., vol. 1, no. 2, Mar. 2017. 